\title{
Production of Cellulose and Profile Metabolites by Fermentation of Glycerol by Gluconacetobacter Xylinus
}

\author{
Francielle Lina Vidotto ${ }^{1}$, Geovana Piveta Ribeiro ${ }^{1}$, Cesar Augusto Tischer ${ }^{*}$. \\ ${ }^{1}$ Universidade Estadual de Londrina - UEL - Departamento de Bioquímica e Biotecnologia - CCE, Londrina, \\ Paraná, Brasil.
}

\begin{abstract}
Because of the widespread occurrence of cellulose in nature, many organisms use glycerol as a source of carbon and energy, so these organisms have drawn attention to the potential use of glycerol bioconversion. The bacteria Gluconacetobacter xylinus, a strictly aerobic strain that performing incomplete oxidation of various sugars and alcohols to cellulose biosynthesis. For this reason, we modify the Hestrim-Schram medium, associating glycerol, glucose and sucrose varying their concentration. The fermentations were performed statically at a temperature of $28^{\circ} \mathrm{C}$ for a period of 10 days. The $\mathrm{pH}$, membrane formation, crystallinity and the production of some metabolites of the $4^{\text {th }}, 7^{\text {th }}$ and $10^{\text {th }}$ days was evaluated. The results showed a higher yield of membrane in the medium containing glucose, gly $1+$ glu2 on 10 fermentation of $3.5 \mathrm{~g} \%$. Through solid -state NMR gave the crystallinity of the membranes, where there was a clear trend toward higher crystallinity membranes with 7 days of fermentation. Metabolic products found in the media by analysis of NMR spectroscopy in liquid were similar, especially for the production of alanine and lactate that were present in all media. The leucine and threonine were present in various media, although in small quantities has been found glutamate.
\end{abstract}

Key Words: Bacterial cellulose, Glycerol, Metabolites, Gluconacetobacter xylinus, Solid state nuclear magnetic resonance, ${ }^{1} \mathrm{H}$ nuclear magnetic ressonance

\footnotetext{
* Author for correspondence: cesar.tischer@me.com, cesar.tischer@uel.br
} 


\section{INTRODUCTION}

Cellulose -(1-4)-D-glucan, is the unbranched polymer composed of dozens of linear glucose units arranged in parallel, forming strongly hydrogen bonds intra and inter regular chain. It is widely distributed in nature, and mainly produced by vascular plants, much of its biosynthesis occurs in plant cell walls, being associated with lignin, hemicellulose and other components 1,2 . Besides plants, many biological organisms distributed in the realms are able to produce this exopolymer as bacteria, fungi, protists, and tunicates. The bacterial cellulose produced by bacteria is chemically pure, free of components such as lignin and hemicellulose, which increase industrial processes due to chemical treatment to remove these components. It is extremely hydrophilic with greater capacity to absorb water compared with the cellulose originated from plants and have a significantly higher degree of polymerization, and can be produced in different substrates and have different shapes. It is endowed with unique physical properties such as ultrafine fibers highly crystalline meshes and high purity, thus, it is a biological material with potential industrial use. These properties combined with a three-dimensional nanostructure provides a wide range of applications ranging from paper and textile industry to the food on the polymer industry ${ }^{3-5}$. Among the bacteria producing it the Gluconacetobacter xylinus species is known as the largest producer ${ }^{6}$, is a gram-

negative, able to grow and produce cellulose from various substrates 7 . A distinct advantage in the study is that its cellulose fibrils are a metabolically inert product, highly pure and of extracellular deposition ${ }^{8,9}$. To produce cellulose many carbon sources may be used, some substrates have been studied such as mannitol, glycerol, fructose, sucrose and galactose seeking to obtain better yields and characteristics of the cellulose fibers. In pursuit of the use of low cost substrates, glycerol is an option, which is the main by-product of biodiesel production and available in plenty currently. Glycerol is a good source of carbon and energy for the growth of various microorganisms, being suitable for biotechnological production of a number of chemicals in fermentative processes $2,10-12$. Beyond the production of cellulose, bioconversion of substrates also generates various metabolites such as dihydroxyacetone, propanediol, succinate, citrate, butanediol, lactate, amino acids,

among others 13,14 . Therefore, studies have been devoted to improve the biotechnological production of cellulose through tests of selected strains, different ways of fermentation and different formulations. The objective of the study was to test the association of glycerol per annum with sources of glucose and sucrose, in order to obtain a cellulose yield and analysis of metabolites formed during fermentation. For the characterization of the products obtained during fermentation techniques such as NMR ${ }^{13} \mathrm{C}$ CP-MAS in liquid and solid state.

\section{MATERIAL AND METHODS}

\section{Bacterial strain and culture medium}

The microorganism used was Gluconacetobacter hansenii ATCC 23769 (former Acetobacter xylinus Yamada) obtained from the André Tosello Foundation of

Campinas, São Paulo-Brazil. The medium used was modified Hestrin-Schramm 15 , wherein the carbon source matched to associations of sources glucose, sucrose and glycerol as follows: glycerol $2 \%+$ glucose $4 \%$, glycerol $1 \%+$ glucose $2 \%$, glycerol $2 \%+8 \%$ sucrose and glucose $8 \%$. 
Cellulose and profile of metabolites

\section{Culture methods}

The fermentation temperature was static at $28^{\circ} \mathrm{C}$ for cooling heating incubator (Sterilifer, São Paulo, Brazil). For each $500 \mathrm{~mL}$ of medium culture was added $4 \mathrm{~mL}$ of standard bacteria, than divided into 10 plates with approximately $40 \mathrm{~mL}$ of medium each. All fermentations lasted for 10 days, and on day 4, 7 and 10 the membranes were removed from the petri dishes and separated from the culture medium.

\section{Treatment of bacterial cellulose}

Obtained celluloses underwent through a cleaning process in $2 \% \mathrm{NaOH}$ solution and then were stored in $1 \% \mathrm{NaOH}$ solution for 48 hours, after this period it were washed in distilled water until neutralization $\underline{16}$.

\section{Yield bacterial cellulose}

Produced cellulose mass was determined by drying and weighing the membranes, dried at a temperature of $80^{\circ} \mathrm{C}$ for 15-30 minutes. After drying, the membranes were weighed on an analytical balance and their yield calculated according to the formula MCB. 100 / Si, where MCB is the dry mass of cellulose and Si the initial amount of sugar present in each plate, adapted from Goelzer and cols ${ }^{16}$.

\section{Solid state NMR and Crystallinity index}

To obtain the crystallinity index it was used the analysis on NMR of solid state, where the membrane fragments were added in $4 \mathrm{~mm}$ rotor and subjected to analyze at CP-MAS NMR probe with rotation of $10 \mathrm{k} \mathrm{Hz}$, using Bruker standard pulse. To calculate the crystallinity index $(\mathrm{CI})$ it was carried out the ratio of the integrated peaks Ic / (Ia + Ic) (x100) to crystalline regions / amorphous regions, peak fit using

Bruker TopSpin 3.1 and Origin 8.1 software ${ }^{16}$.

\section{NMR analysis of the metabolites}

With the liquid medium removed from the plates, centrifuged and dried by lyophilizing, suspended on Deuterium Oxide $99.9 \%$, lyophilized again, and finally solubilized on Deuterium Oxide $99.9 \%$ with $0,312 \mathrm{mM}$ of (trimethyl)propionic-

2,2,3,3-d4 acid (TSP) as chemical shift and concentration reference to metabolites analysis by NMR. Samples were performed in multinuclear $5 \mathrm{~mm}$ BBI probe in quartz tubes NMR. Programs used in the processing of the NMR spectra were on

Chenomx NMR suite, using adapted protocol from Lee ${ }^{17}$ and cols, and examples of the metabolite profiler tool are showed on supplemental material.

\section{RESULTS AND DISCUSSIONS}

\section{Membrane formation and Income}

All fermentations occurred initially at $\mathrm{pH} 5$, by the end of fermentation in medium containing glucose the $\mathrm{pH}$ varied between 3.69 and 5.8, and in medium containing sucrose the lowest $\mathrm{pH}$ was 5.03 and the highest 5.34. The membrane formation began on day 7 of fermentation, medium containing glucose on composition (glu8) was the only that showed it at 4 days of fermentation (Table 1). The membranes obtained with the medium glu8 aspect is thick, swollen and bulky, though has little cellulose mass, corresponding mainly to the water, since this can be absorbing about $100 \mathrm{x}$ its weight in water after produced. The water is retained during the process of biosynthesis, trapped by the fibers that develop around 18,19 . 
Table 1 - Total carbon mass ratio in the medium added with the mass of the membrane obtained for

\begin{tabular}{llll} 
& \multicolumn{2}{c}{ each medium. Yield g\% } \\
\cline { 2 - 4 } Composition & $\mathbf{4}$ & $\mathbf{7}$ & $\mathbf{1 0}$ \\
\hline glu8 & 1.9 & 2.6 & 3 \\
gly2+glu4 & 0 & 0.4 & 1.7 \\
gly2+sac8 & 0 & 0.7 & 1.1 \\
gly1+glu2 & 0 & 1.6 & 3.5 \\
\hline
\end{tabular}

The medium gly1+glu2 had the highest final yield with $3,5 \mathrm{~g} \%$, with a very reasonable income. In a medium containing sucrose, gly $2+\operatorname{sac} 8$, the presence of a thin membrane on day 7 was noticed and day 10 the membrane even thin, occupied the entire area of the petri dish. It was observed that in glu8 a progressive increase of $\mathrm{CB}$, but other medium required a longer time.

\section{Analysis by Nuclear Magnetic Resonance solid (CP MAS ${ }^{13} \mathrm{C} \mathrm{rmn}$ ) of bacterial cellulose membrane}

The cellulose samples were submitted for analysis to compare the crystallinity of the material obtained in different ways (Table 2). The amount of the bacterial cellulose crystallinity index in the literature with glucose as carbon source CI vary between $71 \%$ and $79 \%$. In media containing sucrose crystallinity the rates found were $69 \%$

and $81,2 \% \mathrm{CI}^{20-22}$. Values were mostly similar to those observed in previous studies, between 67 and 79\%, yet an analysis of absolute crystallinity values indicate a clear trend for a higher crystallinity in the membranes obtained with 7 days of fermentation in medium that had glycerol associated, as gly $2+$ glu 4 with $73 \% \mathrm{CI}$ and gly $2+$ sac 8 with $79,4 \%$ CI (Table 2 ). 
Cellulose and profile of metabolites

Table 2 - Crystallinity index of the membranes in culture media containing glycerol.

\begin{tabular}{llll} 
& \multicolumn{3}{l}{ IC\% membrane } \\
\cline { 2 - 4 } Composition & $\mathbf{4}$ & $\mathbf{7}$ & $\mathbf{1 0}$ \\
\hline glu8 & 73 & 67.6 & 71.4 \\
gly2+glu4 & & 73 & 68.5 \\
gly2+sac8 & & 79.4 & 70.4 \\
gly1+glu2 & & 69.9 & 67.6 \\
\hline
\end{tabular}

Relating the crystallinity with the income it can be considered that these characteristics are inversely proportional, as on the 7th day of fermentation crystallinity indexes were higher, membranes yields were low. Considering that the pairing of chains occurs as a phenomenon of post synthesis, from a certain chain length this becomes stationary, pressing the movement of bacteria in the opposite direction to the film formation described by Hesse ${ }^{23}$ with the acronym NOC (nematic ordered cellulose), and results in a less effective pairing of cellulose chains from the CeSa (cellulose synthase) 24,25 . Besides the crystalline regions, cellulose comprises amorphous regions, which depending on the treatment and the cellulose source its degree of crystallinity varies, and its composition determines the type cellulose membrane forming. The literature describes two types of cellulose produced naturally by G. xylinus, the Cel I under normal conditions, and Cel II when subjected to heat stress or mechanical ${ }^{26}$.

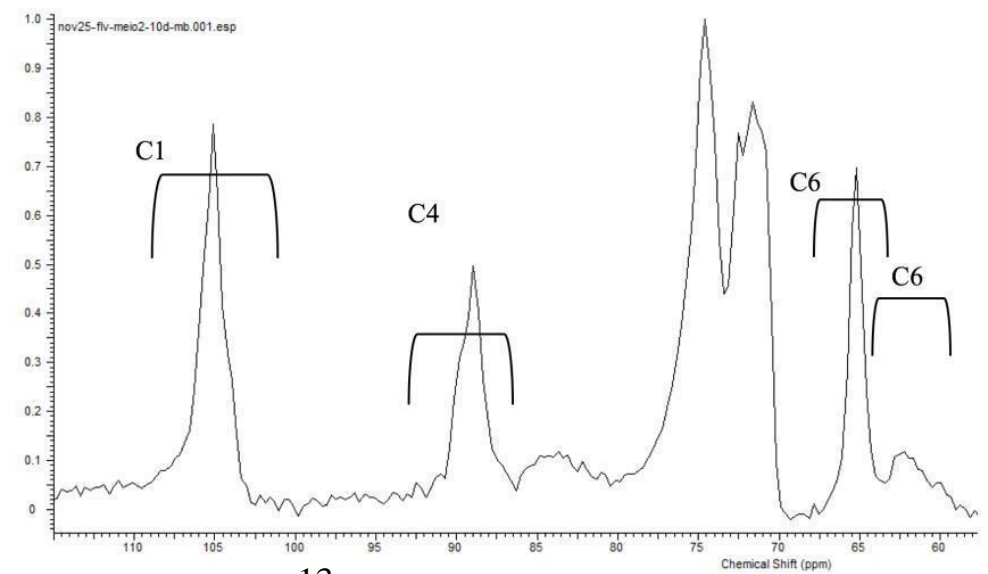

Figure 1 - CP MAS ${ }^{13} \mathrm{C}$ nmr spectrum of bacterial cellulose produced in medium gly $2+\operatorname{sac} 8$ of 10 days fermentation.

Analyses of solid NMR spectra were very similar among themselves, showing characteristic signs for mostly crystalline cellulose type Cel I. The chemical signals obtained exhibit a mixture of $\mathrm{Cel} \mathrm{I} \alpha$ and $\mathrm{I} \beta$ as well as those obtained by Faria-

Tischer $^{26}$, where observed anomeric signal (C1) in the $103 \mathrm{ppm}$ region, the signals for crystalline and amorphous C4 (82-92 ppm) and to C6 between 60 and $65 \mathrm{ppm}$ (Figure 1). Cellulose I is composed of two distinct crystalline phases known as Ia and I $\beta$ which differ by different diffraction patterns, cellulose I (alpha) triclinic and cellulose I (beta) monoclinic. The difference between them is the hydrogen bonds and the cellulose chains frame $5,27,28$. 


\section{Metabolite Analysis by liquid state nuclear magnetic resonance ( $\left.{ }^{1} \mathbf{H} \mathbf{~ n m r}\right)$}

For studies of metabolites and metabolic profiles, analysis by Nuclear Magnetic Resonance (NMR) is an important and advantageous tool due to the ease of

preparation of the samples to be analyzed. In the analysis by ${ }^{1} \mathrm{H}$ nmr in liquid state, it was carried out the quantification of the metabolites present in the media during the 10-day period, where samples were taken on days 4,7 and 10 of fermentation, and all results for metabolites profile are summarized on table 1 of the supplemental material. During fermentation by specific bacteria, G. xylinus, microbial synthesis of cellulose microfibrils is the major metabolite produced, but during this synthesis other metabolites are produced due to microbial metabolism. In addition to

identifying the metabolites, ${ }^{1} \mathrm{H}$ nmr was also used to check the consumption of the carbon sources glucose and sucrose during the fermentation. It was possible to observe a decrease in glucose concentrations on media gly $2+$ glu 4 and gly $1+$ glu 2 on the $4^{\circ}, 7^{\circ}$ and $10^{\circ}$ day of fermentation. Among these, the media glu $2+$ glu 4 had the highest consumption of glucose, due to the participation of glucose in the beginning of biosynthetic pathway to the production of CB and secondary metabolites. Since the media containing sucrose had the presence of glucose, it is due to the breakdown of the disaccharide sucrose into monosaccharides glucose, wherein the media with a higher concentration of glucose, gly $2+\mathrm{sac} 8$, showed high conversion to glucose during the first days of fermentation. In the media gly $2+\operatorname{sac} 8$ sucrose concentration was kept high even on the 10th day of fermentation with $92.5 \%$, this is due to high concentration added in the medium, wherein the microorganism is not able to convert this concentration on the period of 10 days. Most of the identified metabolites were amino acids, and these are synthesized during glycolysis or the citric acid cycle. Alanine is a nonessential amino acid found in all living organisms, synthesized from pyruvate and amino acids with branched side chains such as valine, leucine and isoleucine, are commonly produced by reduction of the pyruvate amination. Alanine was present in all media but in those containing glycerol, concentration of this metabolite was higher and in medium containing only glucose, glu8, its concentration decreased with each passing day. The presence of aspartate and the amino acid lysine and tronina are fully connected as the aspartate, in bacteria, is the precursor of the production of these amino acids. Aspartate was identified only in the medium gly $2+$ glu 4 on day 7 and in the gly $1+$ glu 2 on day 4 , with small concentrations. Leucine was detected by the method in the medium gly 2 + glu 4 where its concentration was higher at day 10 with $4.8 \%$, to the medium gly $1+$ glu2 its highest concentration of $4.2 \%$ was detected on day 4 . To the mediums glu2 + sac8 and glu8, it was not possible to detect the presence of the metabolite. Valine was detected only in the media containing the combination of glycerol and glucose with small concentrations. Threonine was detected in small amounts in almost all media as observed. Its highest concentration was observed in gly $2+$ glu 4 with $2.6 \%$ and other media ranged from 0,4 to $0,7 \%$. Glutamate was identified only on the 7 th day in the medium gly $2+$ glu4. Lysine was also identified only in the medium gly 2 + glu4 on day 7 and 10, with concentrations of $1.1 \%$ and $3.4 \%$. The low presence of this amino acid and aspartate in the media is due to its low concentration, being difficult to identify it. Liu and colleagues ${ }^{29}$ conducted a study analyzing the profile of metabolites produced in agitated and static cultures by G. xylinus by GC-MS analysis, as in this research, they also identified the presence of amino acids alanine, valine and leucine, due to its precursors participate in the metabolic pathway of the microorganism. DHA is a physiological product of the cells involved in the metabolic pathway. The glycolytic pathway uses DHA for ATP generation. The diidroxicetona metabolite is produced by microbial fermentation of glycerol from species of the Acetobacter family ${ }^{30-32}$. The diidroxicetona is one of the metabolites most commonly found in fermentations with glycerol, but found in small quantities 
Cellulose and profile of metabolites

and only in the medium gly $2+\operatorname{sac} 8$ on day 7 with $1.2 \%$ and on day 10 with $1.3 \%$. The presence of lactate in the culture medium was identified by ${ }^{1} \mathrm{H}$ NMR analysis on all media with higher concentration at day 10 in the medium gly $2+\operatorname{sac} 8$ with $14.6 \%$. Studies report the presence of lactate in the fermentation by Enterobacteriaceae and 34 the accumulation of primary metabolites such as acetate and secondary as lactate 33 ,

34 . Traces of propylene glycol were identified among gly $2+\operatorname{sac} 8$ on day 7 with $0.2 \%$. It is described the presence of propylene glycol in studies of gram-negative bacteria using glycerol as substrate 35 .

Principal Component Analysis (PCA) of metabolites

In PCA chart metabolites are represented by vectors, which when suffer decomposition, will be indicated by other vectors in realtion to a main axis component (AC). The vectors that show high correlation with the axis of $\mathrm{CP}$, whether horizontal or vertical, are those considered longer and also are those that

best explain the variability between samples presented in this component 36,37 .
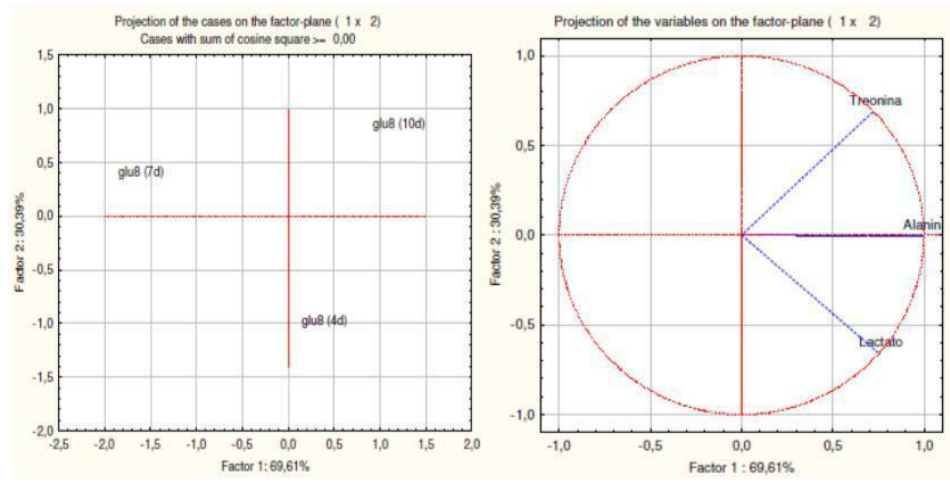

Figure 2 - Projections of production of metabolites through glu8 on day 4, 7 and 10.

Based on the projection of $8 \%$ glucose medium (Figure 5) is possible to observe through the vectors a correlation between days of fermentation and metabolites produced. Only three metabolites have been identified among them being threonine, alanine and lactate. The presence of threonine was favored at day 10 while lactate fermentation on day 4 , but alanine was favored in both fermentation periods.
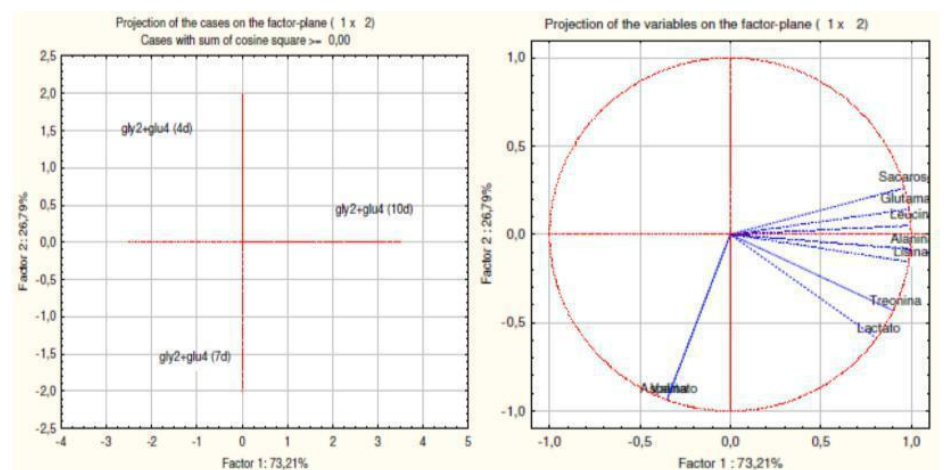

Figure 3 - Projections of production of metabolites through gly $2+$ glu 4 on days 4,7 and 10 .

Metabolites sucrose, alanine, lactate, leucine, lysine and threonine, had their production favoured on day 10 of fermentation on medium gly $2+$ glu 4 . While glutamate and aspartate were favoured on day 7 . On the $4^{\text {th }}$ day of fermentation it was not possible to observe the projections of the vectors due to small concentrations of metabolites produced (Figure 3 ). Projection of medium gly $1+$ glu 2 is shown in 
Figure 4, where vectors show metabolites favouring production on day 10, thus identifying the presence of sucrose, alanine, lactate, leucine and valine. On day 4 metabolites are also produced, but in low concentrations, and it is favoured the production of threonine and aspartate.
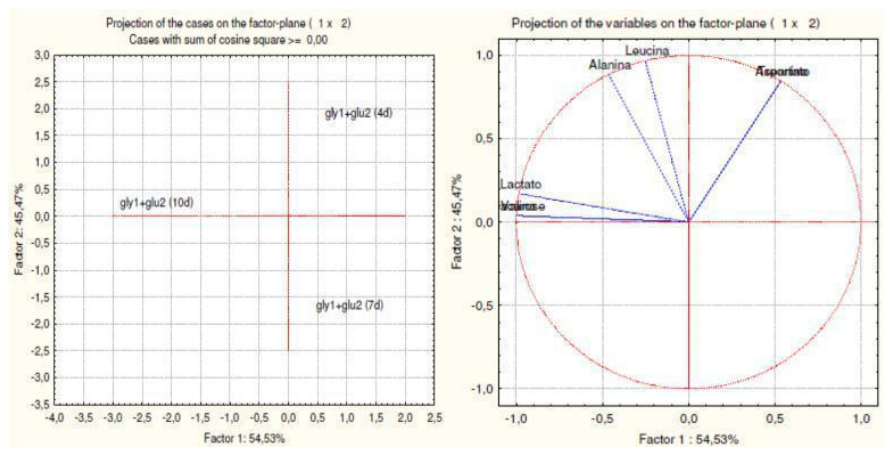

Figura 4 - Projections of production of metabolites through gly $1+$ glu 2 on days 4,7 and 10 .

In the only medium containing glycerol and sucrose (gly2 + sac8) (Figure 5) it is shown that on day 4 of fermentation, small concentrations of metabolites fermentation were produced and the production of lactate and DHA were not favored at this time, but it was positive at day 10 of fermentation. On the 7 th day occurred the production of propylene glycol that did not favor the production of alanine, present in small concentrations.
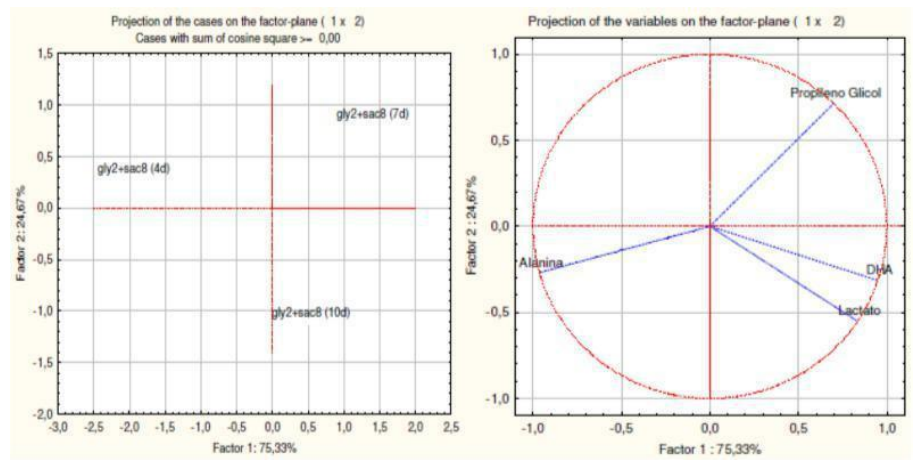

Figure 5 - Projections of production of metabolites through glu $2+\operatorname{sac} 8$ on days 4,7 and 10 .

\section{CONCLUSION}

Glycerol could be used as carbon source for bacterial cellulose production with no loses for yield, crystalline index and type of crystalline arrangement, and gives metabolites with the same profile obtained for the usual glucose medium.

During the production of bacterial cellulose membranes, metabolites formed from the carbon sources glycerol and sucrose gives yield with the medium gly1 + glu2 production rate similar to usual medium.

The bacterial cellulose membranes analyzed by NMR spectroscopy in solid state showed characteristic signals for crystalline celluloses type Cel I. There was a clear tendency for greater crystallinity membranes with 7 days of fermentation with the exceptionally high value for medium gly $2+$ sac 8 with a value of $79,4 \%$ CI. The metabolic products found in the media by analysis of NMR spectroscopy in liquid state were similar, especially the production of alanine and lactate that were present in all media. This way, a research is important in seeking ways to obtain cellulose membranes with low production cost. 
Cellulose and profile of metabolites

\section{ACKNOWLEDGMENTS}

The authors wish to thank the Spectroscopy Facility Laboratory (SPEC-UEL-CT INFRA 2009-01.10.0534.01) at the Universidade Estadual de Londrina. The authors would also like to acknowledge financial support from CNPq-National Counsel of Technological and Scientific Development (447861/2014-0, and 479992/2013-4).

\section{REFERENCES}

1. Delmer DP, Amor Y. CELLULOSE BIOSYNTHESIS. Plant Cell 1995, 7(7): 987-1000.

2. Zhong C, Zhang GC, Liu M, Zheng XT, Han PP, Jia SR. Metabolic flux analysis of Gluconacetobacter xylinus for bacterial cellulose production. Appl. Microbiol. Biotechnol. 2013, 97(14):6189-6199.

3. Lee K-Y, Aitomaki Y, Berglund LA, Oksman K, Bismarck A. On the use of nanocellulose as reinforcement in polymer matrix composites. Compos. Sci. Technol. 2014, 105, 15-27.

4. Klemm D, Heublein B, Fink HP, Bohn A. Cellulose: Fascinating biopolymer and sustainable raw material. Angew. Chem. Int. Ed. 2005, 44(22):3358-3393.

5. Klemm D, Kramer F, Moritz S, Lindstrom T, Ankerfors M, Gray D, Dorris A. Nanocelluloses: A New Family of Nature-Based Materials. Angew. Chem. Int. Ed. 2011, 50(24):5438-5466.

6. Czaja W, Krystynowicz A, Bielecki S, Brown RM. Microbial cellulose - the natural power to heal wounds. Biomaterials 2006, 27(2):145-151.

7. Keshk S, Sameshima K. Evaluation of different carbon sources for bacterial cellulose production. Afr. J. Biotechnol. 2005, 4(6):478-482.

8. Jonas R, Farah LF. Production and application of microbial cellulose. Polym. Degrad. Stab. 1998, 59(1-3):101-106.

9. Mikkelsen D, Flanagan BM, Dykes GA, Gidley MJ. Influence of different carbon sources on bacterial cellulose production by Gluconacetobacter xylinus strain ATCC 53524. J Appl Microbiol 2009, 107 (2):576-583.

10. Heller KB, Lin ECC, Wilson TH. Substrate-specificity and transport-properties of the glycerol facilitator of escherichia-coli. J Bacteriol. 1980, 144 (1):274-278.

11. da Silva GP, Mack M, Contiero J. Glycerol: A promising and abundant carbon source for industrial microbiology. Biotechnol. Adv. 2009, 27 (1):30-39.

12. Wang ZX, Zhuge J, Fang HY, Prior BA. Glycerol production by microbial fermentation: A review. Biotechnol. Adv. 2001, 19(3):201-223.

13. Nabe K, Izuo N, Yamada S, Chibata I. Conversion of glycerol to dihydroxyacetone by immobilized whole cells of acetobacter-xylinum. Appl Environ Microb 1979, 38(6):10561060 .

14. Zhu MM, Lawman PD, Cameron DC. Improving 1,3-propanediol production from glycerol in a metabolically engineered Escherichia coli by reducing accumulation of snglycerol-3-phosphate. Biotechnol Prog 2002, 18(4):694-699.

15. Hestrin S, Schramm M. Synthesis of cellulose by acetobacter-xylinum .2. preparation of freeze-dried cells capable of polymerizing glucose to cellulose. Biochem. J 1954, 58(2):345-352.

16. Goelzer FDE, Faria-Tischer PCS, Vitorino JC, Sierakowski M-R, Tischer CA. Production and characterization of nanospheres of bacterial cellulose from Acetobacter xylinum from processed rice bark. Mater. Sci. Eng., C 2009, 29(2):546-551.

17. Lee J-E, Hwang G-S, Berg FVD, Lee C-H, Hong Y-S. Evidence of vintage effects on grape wines using ${ }^{1}$ H NMR-based metabolomic study. Anal. Chim. Acta 2009, 648(1):7176.

18. Park S, Venditti RA, Jameel H, Pawlak JJ. Changes in pore size distribution during the drying of cellulose fibers as measured by differential scanning calorimetry. Carbohydr. Polym. 2006, 66(1):97-103. 
19. Seifert M, Hesse S, Kabrelian V, Klemm D. Controlling the water content of never dried and reswollen bacterial cellulose by the addition of water-soluble polymers to the culture medium. J. Polym. Sci., Part A: Polym. Chem. 2004, 42(3):463-470.

20. Watanabe K, Tabuchi M, Morinaga Y, Yoshinaga F. Structural features and properties of bacterial cellulose produced in agitated culture. Cellulose 1998, 5(3):187-200.

21. Ruka DR, Simon GP, Dean KM. Altering the growth conditions of Gluconacetobacter xylinus to maximize the yield of bacterial cellulose. Carbohydr. Polym. 2012, 89(2):613622.

22. Mohite BV, Patil SV. Physical, structural, mechanical and thermal characterization of bacterial cellulose by G-hansenii NCIM 2529. Carbohydr. Polym. 2014, 106:132-141.

23. Hesse S, Kondo T. Behavior of cellulose production of Acetobacter xylinum in C-13enriched cultivation media including movements on nematic ordered cellulose templates. Carbohydr. Polym. 2005, 60 (4):457-465.

24. Ross P, Mayer R, Benziman M. Cellulose biosynthesis and function in bacteria. Microbiol Rev 1991, 55(1):35-58.

25. Romling U, Galperin MY. Bacterial cellulose biosynthesis: diversity of operons, subunits, products, and functions. Trends Microbiol 2015, 23(9):545-557.

26. Faria-Tischer PCS, Tischer CA, Heux L, Le Denmat S, Picart C, Sierakowski MR, Putaux JL. Preparation of cellulose II and IIII films by allomorphic conversion of bacterial cellulose I pellicles. Mater. Sci. Eng., C 2015, 51:167-173.

27. Fink HP, Purz HJ, Bohn A, Kunze J. Investigation of the supramolecular structure of never dried bacterial cellulose. Macromol. Symp. 1997, 120:207-217.

28. Koyama M, Helbert W, Imai T, Sugiyama J, Henrissat, B. Parallel-up structure evidences the molecular directionality during biosynthesis of bacterial cellulose. Proc. Natl. Acad. Sci. U.S.A. 1997, 94(17):9091-9095.

29. Liu M, Zhong C, Wu XY, Wei YQ, Bo T, Han PP, Jia SR. Metabolomic profiling coupled with metabolic network reveals differences in Gluconacetobacter xylinus from static and agitated cultures. Biochem. Eng. J. 2015, 101:85-98.

30. Petersen AB, Wulf HC, Gniadecki R, Gajkowska B. Dihydroxyacetone, the active browning ingredient in sunless tanning lotions, induces DNA damage, cell-cycle block and apoptosis in cultured HaCaT keratinocytes. Mutat. Res. Genet. Toxicol. Environ. Mutagen. 2004, 560(2):173-186.

31. Claret C, Salmon JM, Romieu C, Bories A. Physiology of gluconabacter-oxydans during dihydroxyacetone production from glycerol. Appl. Microbiol. Biotechnol. 1994, 41(3):359-365.

32. Hekmat D, Bauer R, Fricke J. Optimization of the microbial synthesis of dihydroxyacetone from glycerol with Gluconobacter oxydans. Bioprocess. Biosyst. Eng. 2003, 26(2):109-116.

33. Homann T, Tag C, Biebl H, Deckwer WD, Schink B. Fermentation of glycerol to 1,3propanediol by klebsiella and citrobacter strains. Appl. Microbiol. Biotechnol. 1990, 33(2):121-126.

34. Murarka A, Dharmadi Y, Yazdani SS, Gonzalez R. Fermentative utilization of glycerol by Escherichia coli and its implications for the production of fuels and chemicals. Appl. Environ. Microbiol. 2008, 74(4):1124-1135.

35. ten Dam J, Hanefeld U. Renewable Chemicals: Dehydroxylation of Glycerol and Polyols. Chemsuschem 2011, 4(8):1017-1034.

36. Yamamoto H, Fujimori T, Sato H, Ishikawa G, Kami K, Ohashi Y. Statistical hypothesis testing of factor loading in principal component analysis and its application to metabolite set enrichment analysis. Bmc Bioinformatics 2014, 15.

37. Cho IH, Kim YS, Lee KW, Choi HK. Determination of differences in the nonvolatile metabolites of pine-mushrooms (Tricholoma matsutake sing.) according to different parts and heating times using H-1 NMR and principal component analysis. J. Microbiol. Biotechnol. 2007, 17(10):1682-1687. 